\title{
An Unusual Case of Entamoeba Histolytica Infection in Miami Fl: Case Report and Review of the Literature
}

\author{
Tahir Maryam, MD, Sendzischew Shane Morgan, MD, Deshpande Amar R, MD, and Milikowski Clara, MD* \\ Pathology and Laboratory Medicine, Jackson Memorial Hospital, University of Miami, USA
}

\begin{abstract}
We report a case of E. histolytica, which is a rare parasitic infection in North America. A 66-year-old Ecuadorian female had an intermittent six-month history of bloating, explosive diarrhea and significant abdominal distension. Histopathological specimen obtained via colonoscopy revealed colitis due to $E$. histolytica. Diagnosis is best accomplished by the combination of serology or antigen testing together with identification of the parasite in the stool or extra-intestinal sites or histologic examination when necessary. With medical tourism and an increase of immigrants from South and Central America, disease endemic to those areas should always be considered.
\end{abstract}

\section{Introduction}

Entamoeba histolytica (EH) infection of the gastrointestinal tract is common in the developing world but rare in North America. The Office of Rare Diseases (ORD) of the National Institutes of Health (NIH) considered it a rare disease. Risk factors for infection include poor sanitation, exposure to contaminated water and sexual or fecal/oral transmission [1]. Liver abscesses are the most frequent complication of invasive amebiasis [2]. Here, we describe a case of an intestinal infection caused by $E$. histolytica in Miami, FL, United States.

\section{Case Report}

A 66-year-old Ecuadoran female presented for an evaluation of abdominal pain and bloody diarrhea. She has chronic colicky lower abdominal discomfort and bloating. She has had colonoscopies since 55 years of age every three years, all of which were reported as unremarkable. In February 2018 she presented to an institution in Ecuador with worsening lower abdominal pain, explosive loose stools 2-3 times daily with blood and mucus. She has no family history of inflammatory bowel disease. Computed Tomography (CT) showed a thickened colon. She underwent colonoscopy in Ecuador and was given the diagnosis of ulcerative colitis. She was started on mesalamine; the rectal bleeding stopped and bowel movements slowed to one per day.

She presented to our institution in July 2018 for a second opinion due to continued intermittent colicky pains, anal discomfort, and straining. Her diarrhea resolved and she was instead experiencing intermittent constipation. She denied viral illness, antibiotic use, ocular inflammation, oral ulcers, or rash.

The differential diagnosis of ulcerative colitis, irritable bowel syndrome and an acute infectious process were considered.
Repeat colonoscopy at our institution showed multiple cecal ulcers with a few tiny sigmoid colon erosions (Figure 1). The cecal biopsies showed colonic mucosa with mild architectural distortion, a dense lymphoplasmacytic and neutrophilic infiltrate in the lamina propia with rare cryptitis. There was a fibrinopurulent exudate which had trophozoites morphologically consistent with E. histolytica (Figure 2). The organisms were approximately 25 microns in diameter, had a prominent nucleus and some had ingested red blood cells (inset). Serology for Entamoeba histolytica IgG was positive, confirming the diagnosis. She was prescribed Metronidazole and Paromomycin.

\section{Discussion}

Intestinal amebiasis caused by the protozoan Entamoeba histolytica is generally asymptomatic and symptomatic patients present with dysentery and extraintestinal disease [3]. Worldwide, approximately 50 million people are affected, with over 100,000 deaths annually [4]. There are four species of intestinal amebae: E. histolytica, E. dispar, E. moshkovskii, and E. Bangladeshi [5]. Most of the symptomatic disease is caused by E. histolytica, and E. dispar is generally considered

\footnotetext{
*Corresponding author: Milikowski Clara, MD., Jackson Memorial Hospital, University of Miami, Pathology and Laboratory Medicine, 1611 NW $12^{\text {th }}$ Ave, Holtz Children's Hospital, $2^{\text {nd }}$ Floor, Suite 2042, FL 33136, Miami, Florida, USA

Accepted: January 25, 2020

Published online: January 27, 2020

Citation: Maryam T, Morgan SS, Deshpande AR, et al. (2020) An Unusual Case of Entamoeba Histolytica Infection in Miami FI: Case Report and Review of the Literature. Res Rev Infect Dis $3(1): 40-43$
} 


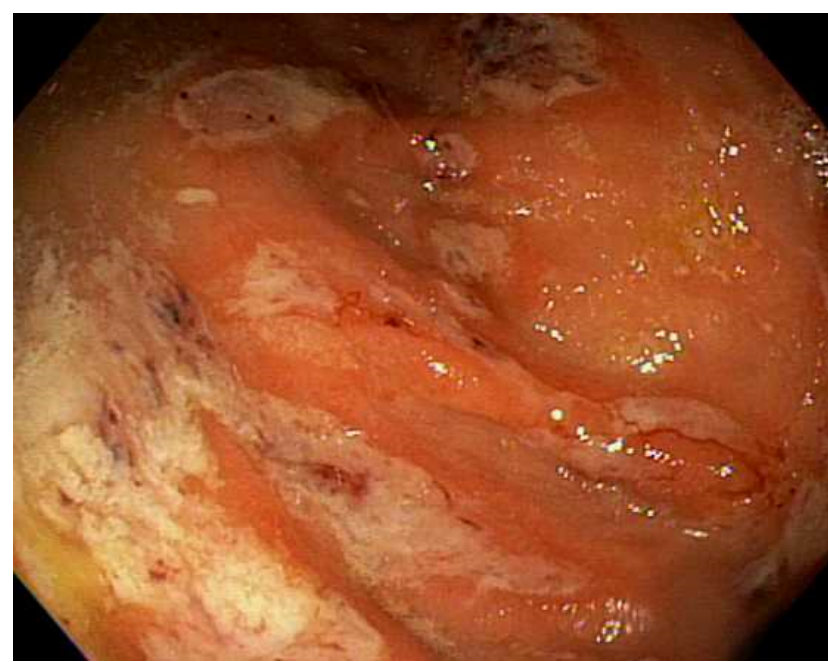

Figure 1: Colonoscopy examination showed multiple cecal ulcers and erosions.

nonpathogenic. Reported infections with E. moshkovskii are becoming more frequent, with increasing evidence of its potential pathogenicity. The pathogenic potential of $E$. Bangladeshi remains unclear [6]. Areas with high rates of amebic infection include India, Africa, Mexico, and parts of Central and South America. The overall prevalence of amebic infection may be as high as 50 percent in some areas [5]. In developed countries, amebiasis is predominantly seen in travelers to endemic areas. In one prospective study of German travelers to the tropics, only 0.3 percent had pathogenic $E$. histolytica infection [7]. Risk factors also include institutionalized patients and homosexuals [1].

In the United States and Europe, homosexual males are principally colonized with nonpathogenic $E$. dispar; in these regions, HIV-infected patients are not considered to be at increased risk for intestinal or extraintestinal amebiasis [8]. There are two parasitic forms, a cyst stage, and a trophozoite stage. Infection occurs following ingestion of amebic cysts via contaminated food or fecal-oral contact [1]. Cysts can remain viable in the environment for months. The ingestion of a single cyst is sufficient to cause the disease. The cysts pass through the stomach to the small intestine, where they excyst to form trophozoites. The trophozoites can then manifest as invasive disease.

The virulence of $E$. histolytica is variable $[9,10]$. Colitis results after penetration of the trophozoite through the intestinal mucus layer, which otherwise acts as a barrier to invasion [3]. The trophozoite can destroy both epithelial cells and inflammatory cells, which is thought to occur through many different mechanisms. Pathogenicity of amebic trophozoites is facilitated by adherence to colonic epithelial cells via a specific lectin [11]. Mammalian cells without $\mathrm{N}$-terminal galactose or $\mathrm{N}$-acetylgalactosamine residues are resistant to adherence by amebic trophozoites, which is consistent with an essential role for the lectin in adhesion. This lectin also plays a role in immunity following colonization. One study from Bangladesh showed that children with a mucosal IgA response against the lec-

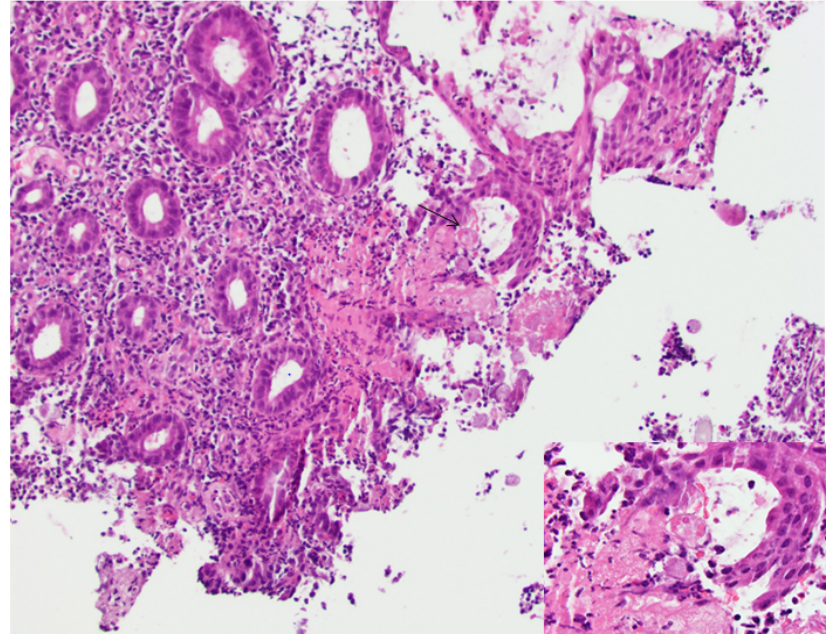

Figure 2: There is an active colitis with organisms within the inflammatory infiltrate (arrow). High-power view of amebic trophozoites shows the distinct cell membrane, foamy cytoplasm, and round, eccentric nucleus with an open chromatin pattern. Ingested red blood cells within the organisms are virtually pathognomonic of E. histolytica. The organisms were approximately 25 microns in diameter, had a prominent nucleus and some had ingested red blood cells (inset).

tin had 86 percent fewer new infections during a one-year period than children without this response $[12,13]$ and, when re-infected, had a lower incidence of symptomatic disease over a four-year follow-up period. Other amebic molecules such as lipophosphopeptidoglycan, peroxiredoxin, arginase, and lysine, and glutamic acid-rich proteins are also implicated in the pathogenesis of amebiasis [14].

Diagnostic techniques include microscopy, antigen detection, serology, molecular techniques, and colonoscopy with histological examination. Culture techniques are limited to research settings. Diagnosis is best accomplished by the combination of serology or antigen testing together with identification of the parasite in the stool or extraintestinal sites.

\section{Stool microscopy}

Stool microscopy can demonstrate cysts or trophozoites but cannot differentiate between $E$. histolytica and the other species. Microscopy requires specialized expertise and is subject to operator error. Organism excretion can vary and a minimum of three specimens on separate days should be sent to detect 85 to 95 percent of infections. Specimens can be concentrated and stained with iodine to detect cysts. For trophozoites, a saline wet mount and a fresh smear stained with iron hematoxylin and Wheatley's trichrome should be performed; fixation with polyvinyl alcohol for delayed staining is often useful.

\section{Antigen testing}

Antigen testing is sensitive, specific, rapid, easy to perform, and can distinguish between $E$. histolytica and $E$. dispar. Stool and serum antigen detection assays that use 
monoclonal antibodies to bind to epitopes present on pathogenic E. histolytica strains (but not on nonpathogenic E. dispar strains) are commercially available for diagnosis of $E$. histolytica infection $[15,16]$. Antigen detection kits using enzyme-linked immunosorbent assay (ELISA), radioimmunoassay, or immunofluorescence have been developed [17-19]. Antigen detection has many advantages, including ease and rapidity of the tests, the capacity to differentiate between strains, greater sensitivity than microscopy, and potential for diagnosis in early infection. The TechLab E. histolytica stool antigen test is an ELISA test that is specific for $E$. histolytica. The assay detects the $E$. histolytica-derived Gal/GalNAc lectin in stool specimen; it has a sensitivity of eighty seven percent and a specificity of $>90$ percent compared with culture [17-19]. A study comparing the TechLab E. histolytica-specific antigen detection test with polymerase chain reaction (PCR) assays showed comparable sensitivities when performed directly on fresh stool specimens [20].

\section{Serology}

Serology is a useful diagnostic tool for amebiasis. Antibodies are detectable within five to seven days of acute infection and may persist for years. Therefore, negative serology is helpful for the exclusion of disease, but positive serology cannot distinguish between acute and previous infection. Indirect hemagglutination (IHA) is the most sensitive serologic assay; it is positive in approximately 90 percent of patients with symptomatic intestinal infection. Agar gel diffusion and counter immunophoresis are less sensitive than IHA. A commercially available ELISA that has a sensitivity of 93 percent compared with IHA has also been developed, $\left(3^{\text {rd }}\right.$ edn, Guerrant R, Walker DH, Weller PF (Eds), Saunders Elsevier, Philadelphia 2011. p.614).

\section{Molecular methods}

Molecular methods to detect DNA or RNA in feces can also be used to differentiate between the three different strains. PCR techniques can detect $E$. histolytica in stool specimens [21]. Studies have shown that PCR is significantly more sensitive than microscopy and that it was 100 percent specific for E. histolytica [22]. PCR is 100 times more sensitive than fecal antigen tests [23]. Many investigators have developed PCR methods for the diagnosis of intestinal amebiasis and differentiation between pathogenic and nonpathogenic amebae [24-26]. These methods are highly sensitive and specific research tools but are generally not commercially available for diagnostic clinical testing [23].

\section{Visual inspection of the colon}

Visual inspection of the colon can be performed by sigmoidoscopy and colonoscopy to make the diagnosis of amebiasis and to exclude other causes of symptoms. However, colonoscopy is not appropriate as a routine diagnostic tool since the presence of amebic ulcerations increase the likelihood of perforation during instillation of air to expand the colon.
Cecum and colon are the most common sites of involvement [27]. The characteristic endoscopic findings of amebic colitis have been reported in the cecum, rectum, ascending colon, transverse colon, sigmoid colon, and descending colon as $93,45,28,25,20$, and 15 percent, respectively [27].

Scrapings or biopsy specimens, best taken from the edge of ulcers, may be positive for cysts or trophozoites on microscopy, and antigen testing for E. histolytica may be positive. Colonic lesions in amebic dysentery range from nonspecific mucosal thickening and inflammation to classic flask-shaped amebic ulcers.

The differential diagnosis of $E$. histolytica amebiasis includes other causes of acute diarrhea or bloody stools, particularly bacterial pathogens including Shigella, Escherichia coli, Salmonella, Campylobacter, Clostridium difficile, and some Vibrio species. Noninfectious etiologies include ischemic bowel and inflammatory bowel disease.

Invasive colitis is treated with metronidazole (alternative therapies include tinidazole, ornidazole, and nitazoxanide), followed by a luminal agent (such as paromomycin, diiodohydroxyquin, or diloxanide furoate) to eliminate intraluminal cysts. A 10-day course of metronidazole eliminates intraluminal infection in many cases, but a second agent is still warranted, (Drugs for Parasitic Infections, $3^{\text {rd }}$ edn, The Medical Letter, New Rochelle, NY 2013) [28].

Prevention of amebic infection in travelers to endemic areas involves avoidance of untreated water in endemic areas and uncooked food, such as fruit and vegetables that may have been washed in local water. Amebic cysts are resistant to chlorine at the levels used in water supplies, but disinfection with ultraviolet light are adequate. Development of both parenteral and oral vaccines for humans is in progress [28].

\section{Summary}

In summary, we report a case of E. histolytica in a developed country. Travel history or country of origin is important pieces of clinical information to obtain. Amebiasis occurs worldwide; the prevalence is disproportionately higher in developing countries because of poor socioeconomic conditions and sanitation levels. However, sporadic cases can occur in the developed countries. With medical tourism and immigration from endemic countries, amebiasis should be considered in the differential diagnosis in patients presenting with symptoms from endemic areas. Clinically amebiasis generally has a subacute onset, usually over one to three weeks. Diagnosis is best accomplished by the combination of serology or antigen testing together with identification of the parasite in the stool or extraintestinal sites (such as liver abscess pus). On rare instances, as in this case, an evaluation of the tissue biopsy is considered on a high index of suspicion and inconclusive serology or antigen testing.

\section{Conflict of Interest Statement}

The authors declare no conflict of interest. 


\section{References}

1. Salit IE, Khairnar K, Gough K, et al. (2009) A possible cluster of sexually transmitted Entamoeba histolytica: Genetic analysis of a highly virulent strain. Clin Infect Dis 49: 346-353.

2. Rao S, Solaymani MS, Petri WA Jr, et al. (2009) Hepatic amebiasis: A reminder of the complications. Curr Opin Pediatr 21: 145149.

3. Haque R, Huston CD, Hughes M, et al. (2003) Amebiasis. N Engl J Med 348: 1565-1573.

4. Bercu TE, Petri WA, Behm JW (2007) Amebic colitis: New insights into pathogenesis and treatment. Curr Gastroenterol Rep 9: 429-433.

5. Parija SC, Mandal J, Ponnambath DK (2014) Laboratory methods of identification of Entamoeba histolytica and its differentiation from look-alike Entamoeba spp. Trop Parasitol 4: 90-95.

6. Royer TL, Gilchrist C, Kabir M, et al. (2012) Entamoeba bangladeshi nov. sp., Bangladesh. Emerg Infect Dis 18: 1543-1545.

7. Weinke T, Friedrich JB, Hopp P, et al. (1990) Prevalence and clinical importance of Entamoeba histolytica in two high-risk groups: Travelers returning from the tropics and male homosexuals. J Infect Dis 161: 1029-1031.

8. Moran P, Ramos F, Ramiro M, et al. (2005) Infection by human immunodeficiency virus-1 is not a risk factor for amebiasis. Am J Trop Med Hyg 73: 296-300.

9. Padilla-Vaca F, Anaya-Velazquez F (2010) Insights into Entamoeba histolytica virulence modulation. Infect Disord Drug Targets 10: 242-250.

10. Mortimer L, Chadee K (2010) The immunopathogenesis of Entamoeba histolytica. Exp Parasitol 126: 366-380.

11. Stanley SL Jr (2003) Amoebiasis. Lancet 361: 1025-1034.

12. Haque R, Duggal P, Ali IM, et al. (2002) Innate and acquired resistance to amebiasis in bangladeshi children. J Infect Dis 186: 547-552.

13. Haque R, Mondal D, Duggal P, et al. (2006) Entamoeba histolytica infection in children and protection from subsequent amebiasis. Infect Immun 74: 904-909.

14. Lejeune M, Rybicka JM, Chadee K (2009) Recent discoveries in the pathogenesis and immune response toward Entamoeba histolytica. Future Microbiol 4: 105-118.
15. Rayan HZ (2005) Microscopic overdiagnosis of intestinal amoebiasis. J Egypt Soc Parasitol 35: 941-951.

16. Gonzalez-Ruiz A, Haque R, Rehman T, et al. (1992) Further diagnostic use of an invasive-specific monoclonal antibody against Entamoeba histolytica. Arch Med Res 23: 281-283.

17. Haque R, Kress K, Wood S, et al. (1993) Diagnosis of pathogenic Entamoeba histolytica infection using a stool ELISA based on monoclonal antibodies to the galactose-specific adhesin. J Infect Dis 167: 247-249.

18. Haque R, Ali IK, Akther S, et al. (1998) Comparison of PCR, isoenzyme analysis, and antigen detection for diagnosis of Entamoeba histolytica infection. J Clin Microbiol 36: 449-452.

19. Spadafora L, Kearney MR, Siddique A, et al. (2016) Species-specific immunodetection of an Entamoeba histolytica cyst wall protein. PLoS Negl Trop Dis 10: e0004697.

20. Haque R, Petri WA (2006) Diagnosis of amebiasis in Bangladesh. Arch Med Res 37: 273-276.

21. Qvarnstrom $Y$, James $C$, Xayavong $M$, et al. (2005) Comparison of real-time PCR protocols for differential laboratory diagnosis of amebiasis. J Clin Microbiol 43: 5491-5497.

22. Madison AS, Relich RF, Doyle L, et al. (2016) Multicenter evaluation of BD Max enteric parasite real-time PCR assay for detection of Giardia duodenalis, Cryptosporidium hominis, Cryptosporidium parvum, and Entamoeba histolytica. J Clin Microbiol 54: 2681-2688.

23. Fotedar R, Stark D, Beebe N, et al. (2007) Laboratory diagnostic techniques for Entamoeba species. Clin Microbiol Rev 20: 511-532.

24. Liang SY, Hsia KT, Chan YH, et al. (2010) Evaluation of a new single-tube multiprobe real-time PCR for diagnosis of Entamoeba histolytica and Entamoeba dispar. J Parasitol 96: 793-797.

25. Hamzah Z, Petmitr S, Mungthin M, et al. (2010) Development of multiplex real-time polymerase chain reaction for detection of Entamoeba histolytica, Entamoeba dispar, and Entamoeba moshkovskii in clinical specimens. Am J Trop Med Hyg 83: 909-913.

26. Tanyuksel M, Petri WA (2003) Laboratory diagnosis of amebiasis. Clin Microbiol Rev 16: 713-729.

27. Horiki N, Furukawa K, Kitade T, et al. (2015) Endoscopic findings and lesion distribution in amebic colitis. J Infect Chemother 21: 444-448.

28. Lotter H, Tannich E (2006) The current status of an amebiasis vaccine. Arch Med Res 37: 292-296.

DOI: $10.36959 / 719 / 561$

Copyright: (c) 2020 Maryam T, et al. This is an open-access article distributed under the terms of the Creative Commons Attribution License, which permits unrestricted use, distribution, and reproduction in any medium, provided the original author and source are credited. 